\title{
Allgemeine Rahmenbedingungen und Erläuterungen zum Arbeitsvertrag
}

Aufgrund der seit 1991 kantonal von den Ärztegesellschaften ausgearbeiteten Lohnempfehlungen für Medizinische Praxisassistentinnen werden keine gesamtschweizerischen Lohnempfehlungen mehr publiziert.

Bei individuellen Lohnverhandlungen sollen für Medizinische Praxisassistentinnen die nachstehend aufgeführten Rahmenbedingungen berücksichtigt werden, nämlich:

\section{Ausbildung}

- Diplom der Verbindung der Schweizer Ärzte (Vignette) bzw. Fähigkeitsausweis der FMH inkl. Strahlenschutzausweis bzw. Röntgenbewilligung

- Eidg. Fähigkeitszeugnis Medizinische Praxisassistentin

2. Arbeitsbedingungen: Berechnungsgrundlagen

- 42-Stunden-Woche im Jahresdurchschnitt

- 4 Wochen Ferien (Medizinische Praxisassistentinnen unter 20 und ab 50 Jahren: 5 Wochen) Nennenswerte Abweichungen von diesen Bedingungen können auf den Lohn umgerechnet werden.

\section{Regionale Gegebenheiten}

Die Löhne sollen den regionalen Gegebenheiten angepasst werden.

\section{13. Monatslohn}

Am Jahresende ist der Medizinischen Praxisassistentin ein 13. Monatslohn auszurichten. Umfasst das Arbeitsverhältnis nicht das ganze Kalenderjahr, so ist er anteilmässig zu bezahlen.

5. Dienstalterszulagen und Reallohnerhöhungen Die Höhe einer Dienstalterszulage soll auch für 2015 ein Thema des jährlichen Qualifikationsgespräches bilden. Insbesondere soll bei der Festlegung des Lohnes auch jede zusätzliche Verantwortung (z.B. Ausbilden von Lernenden) oder Weiterbildung (z.B. Dosisintensives Röntgen usw.) der Angestellten berücksichtigt und der Lohn dementsprechend angepasst werden.

Korrespondenz: FMH /Abteilung MPA Elfenstrasse 18 CH-3000 Bern 15 Tel. 0313591111 Fax 0313591112
6. Teuerungsausgleich (www.bfs.admin.ch)

Die Teuerung soll ausgeglichen werden. Wo der Teuerungsausgleich vertraglich vereinbart ist, muss dieser auf jeden Fall gewährt werden. Im Falle einer Minusteuerung ist keine Teuerung auszugleichen (LIKP September 2014: -0,1\%, Oktober 2014: 0,0\%, Indexbasis Dez. 2010).

\section{Teilzeitarbeit im Monatslohn}

Bei Teilzeitarbeit beträgt der Bruttolohn (bei 42 Wochenstunden als Berechnungsgrundlage) 1/42 eines vollen Monatslohnes, multipliziert mit der Anzahl der vereinbarten Wochenarbeitsstunden.

\section{Stundenlohn}

Bei sehr geringer und gleichzeitig unregelmässiger Arbeitszeit empfiehlt sich die Ausrichtung eines Stundenlohnes. Als Stundenlohnansatz empfehlen wir 6\%o eines Monatslohnes für ein Vollzeitpensum (13. Monatslohn ist anteilmässig darin enthalten). Zusätzlich muss auf diesem Ansatz ein Ferienanteil von 8,33\% ausgerichtet werden, der den üblichen 4 Wochen Ferien entspricht und auf jeder Lohnabrechnung separat auszuweisen ist (bei 5 Wochen Ferien 10,64\%, bei 6 Wochen Ferien 13,04\% usw.). Diese Berechnung gilt auch bei der Auszahlung von Überstunden.

\section{9. Überstunden (Ziff. 2 des Mustervertrags)}

Wenn immer möglich, sollen die Überstunden durch Ferien oder Freizeit gleicher Dauer ausgeglichen werden. Ist dies nicht möglich, kann neu gewählt werden zwischen folgenden Varianten:

- Entschädigung samt einem Lohnzuschlag von 25\% für Vollzeit- und Teilzeitangestellte (Vorzugsvariante FMH und BSMPA).

- Bei Teilzeitarbeit werden Überstunden, so lange sie zusammen mit dem Teilzeitpensum die betriebsübliche Arbeitszeit für eine Vollzeitangestellte nicht überschreiten, lediglich nach dem normalen Stundenansatz vergütet (Vorzugsvariante SVA).

- Arbeitgeber und Med. Praxisassistentin können durch schriftliche Vereinbarung eine andere Regelung wählen.

Als Stundenlohnansatz gelten 6\%o eines Monatslohns für ein Vollzeitpensum zuzüglich Ferienanteil (vgl. Ziff. 8 Stundenlohn).

\section{Für alle vereinbarten Löhne gelten folgende} Bestimmungen und Empfehlungen:

1. Abzüge vom Bruttolohn

- AHV, IV, EO, ALV: 6,25\% (AHV, IV, EO = 5,15\%, $\mathrm{ALV}=1,1 \%)$ 


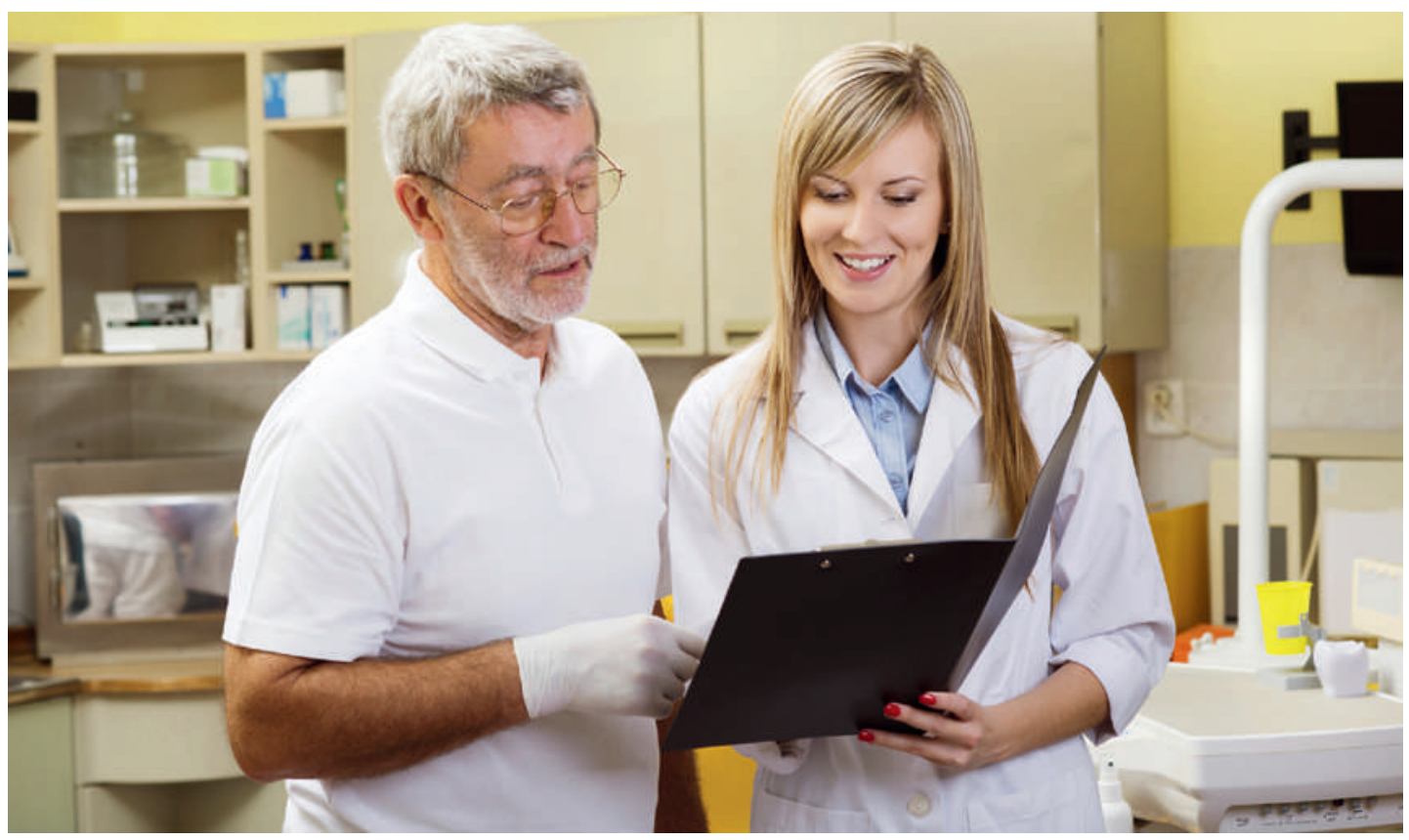

Wenn möglich sollen Überstunden durch Ferien oder Freizeit gleicher Dauer ausgeglichen werden.

- Nichtberufsunfallversicherung (bei einer wöchentlichen Arbeitszeit von mehr als 8 Stunden): Abzug gemäss konkretem Versicherungsvertrag

- Berufliche Altersvorsorge (2. Säule BVG): Arbeitnehmeranteil (normalerweise 50\%) des altersabhängigen Beitrages gemäss Versicherungsausweis.

\section{Arbeitsverträge und weitere Anstellungs-} bedingungen

Wir empfehlen nachdrücklich die schriftliche Vertragsform auf dem von der FMH und den Berufsverbänden der Medizinischen Praxisassistentinnen gemeinsam erarbeiteten Vertragsformular mit zugehörigen kantonalen Empfehlungen; Bezugsquellen:

- Verbindung der Schweizer Ärztinnen und Ärzte FMH, Elfenstrasse 18, Postfach 300, 3000 Bern 15, Tel. 03135911 11, www.fmh.ch, E-Mail: mpa[at] fmh.ch

- Association Romande des Assistantes Médicales ARAM, 1003 Lausanne, Tel. 0793801244 / 07743679 27, www.aram-vd.ch, E-Mail: info[at] aram-vd.ch

- Berufsverband Medizinischer Praxisassistentinnen BMPA, Obergrundstrasse 65, 6003 Luzern, Tel. 04131011 21, www.bmpa.ch, E-Mail: sekretariat[at]bmpa.ch

- Bund Schweizer Verbände Medizinischer PraxisAssistentinnen BSMPA, Postfach, 2051 Biel, Tel. 07967454 71, www.bsmpa.ch, E-Mail sekretariat[at]bsmpa-fsaam.ch

- Schweiz. Verband Medizinischer PraxisAssistentinnen SVA, Postfach 6432, 3001 Bern, Tel. 031
38054 54, www.sva.ch, E-Mail: sekretariat[at] sva.ch

- Association genevoise des assistantes médicales (AGAM), 1200 Genève, www.agam-ge.ch, E-Mail: presidente[at]agam-ge.ch

Der Berufsverband der Medizinischen Praxisassistentinnen im Tessin hat eine eigene Arbeitsvertragskonvention resp. Arbeitsvertrag; Bezugsquelle:

- Assoziazione Ticinese Assistenti di studio Medico (ATAM), c/o M. Carrera, Via dei Faggi 2 A, 6912 Lugano-Pazzallo, www.atam.ch, E-Mail: atam[at] bluewin.ch

Die Löhne für Lernende richten sich nach den kantonalen Empfehlungen.

FMH-Delegierte für MPA-Fragen:

Dr. med. Adrian Sury, Präsident / Dr. med. Renato Tognina, Vizepräsident

Bund Schweizer Verbände Medizinischer PraxisAssistentinnen BSMPA (AGAM, ATAM, BMPA): Elwina Kaufmann, Präsidentin BSMPA

Association Romande des Assistantes Médicales ARAM:

Anne-Claude Perrette und Marie-Paule Fauchère, Präsidentinnen

Schweizerischer Verband Medizinischer PraxisAssistentinnen SVA:

Fürsprecher Bruno Gutknecht, Zentralsekretär 